\title{
The cobordism class of the multiple points of immersions
}

\author{
GÁBOR BRAUN
}

\begin{abstract}
Using generating functions, we derive a multiple point formula for every generic immersion $f: M^{m} \rightarrow N^{n}$ between even dimensional oriented manifolds. This produces explicit formulas for the signature and Pontrjagin numbers of the multiple point manifolds. The formulas take a particular simple form in many special cases, for example, when $f$ is nullhomotopic, we recover Szúcs's formulas in [3]. They also include Hirzebruch's virtual signature formula [1, 9.3(4')].
\end{abstract}

57R20, 57R42; 57R75, 16W60

\section{Introduction}

Let $f: M^{m} \hookrightarrow \rightarrow N^{n}$ be a generic immersion between oriented compact even dimensional smooth manifolds.

Aims Let $M^{\times k}$ be the cartesian product of $k$ copies of $M$ and $f^{\times k}: M^{\times k} \rightarrow N^{\times k}$ be the map induced by $f$ between the products. Let

$$
\Delta:=\{(x, x, \ldots, x): x \in N\} \subseteq N^{\times k}
$$

be the (narrow) diagonal in $N^{\times k}$. Then the $k$-tuples of the multiple points of $f$ form the manifold

$(1-1) \quad \widetilde{\Delta}_{k}(f):=\left\{\left(x_{1}, \ldots, x_{k}\right) \in\left(f^{\times k}\right)^{-1}(\Delta): x_{i} \neq x_{j}\right.$ if $\left.i \neq j\right\}$.

Note that by permuting the coordinates, the symmetric group $S_{k}$ acts on $\widetilde{\Delta}_{k}(f)$. Factoring out with the action leads to the manifold of $k$-tuple points of $f$ :

$$
\Delta_{k}(f):=\widetilde{\Delta}_{k}(f) / S_{k} .
$$

If the action of $S_{k}$ on $\widetilde{\Delta}_{k}(f)$ preserves orientation (ie $n-m$ is even or $k=1$ ), then the factor $\Delta_{k}(f)$ is naturally oriented.

We are going to express the signature and the characteristic numbers of the manifold of $k$-tuple points of $f$ in terms of cohomological invariants of $M, N$ and $f$ (formulas $(2-4),(2-6)$ and $(2-10))$. 
Special cases Our formulas are particularly simple in many special cases as shown in Subsection 2.3. For example, if $N$ is a sphere, we recover Szúcs's formulas (here (2-20) and (2-21)) from [3, Theorems 4 and 5].

Our formula also generalizes Hirzebruch's virtual signature formula, which we recall now.

Let $f: M \rightarrow N$ be a union of embeddings of codimension 2 manifolds $M_{1}, \ldots, M_{k}$ into $N$. Recall that every codimension 2 embedded manifold is the set of zeros of a transversal section of a 2 dimensional vector bundle. Let $V_{i}$ be such a bundle for $M_{i}$, and let us denote the Euler class of this bundle by $e_{i}$. The manifold $\Delta_{k}(f)$ of $k$-tuple points of $f$ is exactly the intersection of the submanifolds $M_{1}, \ldots, M_{k}$. Hirzebruch's virtual signature formula from $\left[1,9.3\left(4^{\prime}\right)\right]$ states that its signature is

$$
\sigma\left(\Delta_{k}(f)\right)=\left\langle L(N) \prod_{i=1}^{k} e_{i} L\left(V_{i}\right)^{-1},[N]\right\rangle,
$$

where $L(N)$ is the Hirzebruch class of the tangent bundle of $N$ and, similarly, $L\left(V_{i}\right)$ is the Hirzebruch class of $V_{i}$.

Hirzebruch uses "index" for "signature" and also uses a different notation. We can get back Hirzebruch's original formula by replacing in the above formula $\sigma$ with $\tau$, the number $k$ with $r$, the manifold $\Delta_{k}(f)$ with $V^{n-2 r}$, the manifold $N$ with $M$, the expression $\langle-,[N]\rangle$ with $\varkappa^{n}[-]$, the class $L(N)$ with $\sum_{i=0}^{\infty} L_{i}\left(p_{1}\left(M^{n}\right), \ldots, p_{i}\left(M^{n}\right)\right)$, and $e_{i} L\left(V_{i}\right)^{-1}$ with $\tanh v_{i}$. All but the last replacement are just changes in notation. To justify the last replacement, note that the dual class $v_{i}$ of $M_{i}$ is the Euler class $e_{i}$ of $V_{i}$ and hence

$$
e_{i} L\left(V_{i}\right)^{-1}=e_{i} \frac{\tanh e_{i}}{e_{i}}=\tanh e_{i}=\tanh v_{i}
$$

Our Corollary 2.3 generalizes Hirzebruch's formula to generic immersions with even codimension by introducing the cohomology class $B_{k}(f)$ in (2-12) such that for any immersion $f: M \leftrightarrow N$, we get:

$$
\sigma\left(\Delta_{k}(f)\right)=\frac{1}{k !}\left\langle L(N) B_{k}(f),[N]\right\rangle .
$$

When $M$ is the disjoint union of manifolds $M_{i}$, each of which is embedded by $f$, then $B_{k}(f)$ factors into a product of some classes of the $M_{i}$ (see Theorem 2.4). If all $M_{i}$ has codimension 2, then this formula reduces to Hirzebruch's (1-2) (see the discussion after Theorem 2.4). 
Equation (1-4) has a version in (2-10) using the cohomology of $M$ instead of the cohomology of $N$. Szúcs obtained this version in the special case when $N$ is a Euclidean space in [3, Theorem 4], which we reproduce as (2-20).

The main idea Using the definition in (1-1), let $i_{k}: \widetilde{\Delta}_{k}(f) \rightarrow M^{\times k}$ denote the inclusion and $j_{k}: \widetilde{\Delta}_{k}(f) \rightarrow M$ the projection to the first coordinate. What we essentially do is deduce an explicit formula for $j_{k !} i_{k}^{*}$ (Theorem 2.2). The formulas for the characteristic numbers are applications of this formula.

How do we compute $j_{k !} i_{k}^{*}$ ? First, in Section 4, we apply Ronga's Clean Intersection Theorem [2, Proposition 2.2] (repeated here as Theorem 4.2) to obtain the recursion (4-1) for this map. Then in Section 5 we interpret this recursion using generating functions, which produces an easy way to solve it.

Acknowledgements This work was partially supported by Hungarian National Research Fund, grant No T 042 769. The author thanks András Szúcs for his encouragement and support.

\section{Main results}

\subsection{Notation}

We fix our notation for the rest of the paper. Let $f: M^{m} \rightarrow N^{n}$ be a generic immersion of compact oriented smooth manifolds with even codimension. The dimension of the components of the manifolds need not be the same. Let $m$ be the dimension function of $M$ which maps every component of $M$ to its dimension, and, similarly, let $n$ be the dimension function of $N$. Now, $f$ having even codimension means that for every $x \in M$ the number $n(f(x))-m(x)$ is even.

Cohomology classes We fix notation for some cohomology classes. Let $v$ be the normal bundle of $f$ and let $e=e(v)$ be the Euler class of $v$. Let $L(\xi)$ be the Hirzebruch class of the bundle $\xi$. For a manifold $X$, we write $L(X)$ for $L(T X)$, the Hirzebruch class of the tangent bundle of $X$. Similarly, we define $P(\xi)$ to be the total Pontrjagin class of $\xi$, and let $P(X):=P(T X)$ be the total Pontrjagin class of the manifold $X$. We use similar notation for Chern classes with $c$ instead of $P$.

We need further notation from [3]. Let $J=\left(j_{1}, j_{2}, \ldots, j_{l}\right)$ be a sequence of nonnegative integers. For a total cohomology class $a$, let $a_{i}$ be its $i$ dimensional part, and let $a_{J}:=a_{j_{1}} a_{j_{2}} \cdots a_{j_{l}}$. For example, if $X$ is a compact manifold, then $p_{J}[X]:=$ 
$\left\langle P(X)_{J},[X]\right\rangle$ is the Pontrjagin number of $X$ corresponding to $J$ (assuming that $\sum j_{i}$ is the dimension of $X$ ).

We write cup products either as ordinary products or with the symbol $\cup$ for the operation. We use $\times$ for cross products.

Equivalence relations Equivalence relations naturally arise in our treatment; see Section 4 or Szúcs [3], which is the starting point of our investigation.

Let $\operatorname{Eq}(k)$ be the set of all equivalence relations on $\{1, \ldots, k\}$. We will think of an equivalence relation $\alpha$ as the set of its equivalence classes, thus $\Theta \in \alpha$ will denote that $\Theta$ is an equivalence class of $\alpha$. Every $\Theta \in \alpha$ is a subset of $\{1, \ldots, k\}$ and so $\Theta$ is ordered by the usual ordering on integers. Moreover, there is an ordering among the equivalence classes themselves: $\Theta_{1}<\Theta_{2}$ if and only if the smallest element of $\Theta_{1}$ is smaller than the smallest element of $\Theta_{2}$. Hence every equivalence relation $\alpha$ is the ordered set of its equivalence classes.

Let $\alpha[i]$ be the equivalence class of $\alpha$ containing $i$. In particular, $\alpha[1]$ is the smallest equivalence class of $\alpha$.

We will let $0=0(k)$ denote the trivial equivalence relation, under which different elements are not equivalent. Let $1=1(k)$ denote the universal equivalence relation, under which all elements are equivalent.

Whenever we write $\prod_{\Theta \in \alpha}$ we assume that the terms of the product appear in the order determined by the ordering of $\alpha$. Similar remark applies to $\prod_{i \in \Theta}$ and other products with ordered index set.

We will denote by $|X|$ the number of elements of the set $X$. For example, $|\alpha|$ is the number of equivalence classes of $\alpha$.

Maps We list the maps between topological spaces we will use in our formulas. These are variants of the diagonal map and the graph of $f^{\times k}$. Below $k$ is a positive integer and $\alpha$ is an equivalence relation on $\{1, \ldots, k\}$. Moreover, $x_{i}$ denotes the $i$-th coordinate of $x$. For an element $x$ of $M^{\times|\alpha|}$ and $\Theta \in \alpha$, let $x_{\Theta}$ denote the $\Theta$-coordinate of $x$. We define $x_{\Theta}$ similarly for $M \times N^{\times(|\alpha|-1)}$, where $M$ is the $\alpha[1]$-coordinate and the 
other coordinates are identified with the other classes of $\alpha$.

$$
\begin{array}{rlrl}
\Delta^{1(k)}=\Delta_{k}: M \rightarrow M^{\times k}, & \Delta_{k}(x)_{i}:=x & \\
\Delta^{\alpha}: M^{\times|\alpha|} \rightarrow M^{\times k}, & \Delta^{\alpha}(x)_{i}:=x_{\alpha[i]} \\
\Gamma^{1(k)}=\Gamma_{k}: M \rightarrow M \times N^{\times(k-1)}, & \Gamma_{k}(x)_{i}:= \begin{cases}x & \text { if } i=1 \\
f(x) & \text { if } i>1\end{cases} \\
\Gamma^{\alpha}: M \times N^{\times(|\alpha|-1)} \rightarrow M \times N^{\times(k-1)}, & \Gamma^{\alpha}(x)_{i}:= \begin{cases}f\left(x_{\alpha[i]}\right) & \text { if } \alpha[i]=\alpha[1] \\
x_{\alpha[i]} & \text { and } i>1\end{cases}
\end{array}
$$

Note that if $M=N$ and $f$ is the identity, then $\Gamma^{\alpha}=\Delta^{\alpha}$. Occasionally, we will use $\Delta_{k}$ for manifolds other than $M$. The context will always make this clear.

We need two maps from the multiple point manifold $\widetilde{\Delta}_{k}(f)$ defined in (1-1): the canonical inclusion $i_{k}: \widetilde{\Delta}_{k}(f) \rightarrow M^{\times k}$ and the projection $j_{k}: \widetilde{\Delta}_{k}(f) \rightarrow M$ to the first coordinate of $M^{\times k}$. When we want to include $f$ in the notation, we write $i_{k}^{(f)}$ and $j_{k}^{(f)}$.

Abbreviation To make formulas more readable, we introduce a shorthand notation for a frequent constant:

$$
A_{k}:=(-1)^{k-1}(k-1) !
$$

\subsection{The general formula}

Now we state our main results, which will be proved in later sections.

Let $f: M^{m} \rightarrow N^{n}$ be a generic immersion of oriented compact manifolds with even codimension. We start with the general formula for signature and characteristic numbers:

Theorem 2.1 The signature and the Pontrjagin numbers of $\Delta_{k}(f)$ are

$$
\begin{aligned}
\sigma\left(\Delta_{k}(f)\right) & =\frac{1}{k !}\left\langle j_{k !} i_{k}^{*}\left(L(M) \times \searrow_{i=2}^{k} L(v)^{-1}\right),[M]\right\rangle \\
& =\frac{1}{k !}\left\langle L(N) f_{!} j_{k !} i_{k}^{*}\left(\searrow_{i=1}^{k} L(v)^{-1}\right),[N]\right\rangle, \\
p_{J}\left[\Delta_{k}(f)\right] & =\frac{1}{k !}\left\langle j_{k !} i_{k}^{*}\left(P(M) \times \searrow_{i=2}^{k} P(v)^{-1}\right)_{J},[M]\right\rangle .
\end{aligned}
$$


If $M$ and $N$ are almost complex, then we have a similar formula for Chern numbers:

$$
c_{J}\left[\Delta_{k}(f)\right]=\frac{1}{k !}\left\langle j_{k !} i_{k}^{*}\left(C(M) \times \searrow_{i=2}^{k} C(v)^{-1}\right)_{J},[M]\right\rangle .
$$

Formulas (2-4) and (2-5) can also be written in the form analogous to (2-3).

To make these formulas explicit, we have to compute $j_{k} i_{k}^{*}$ (or $f_{!} j_{k} ! i_{k}^{*}$ ). Recall that $e=e(v)$ is the Euler class of the normal bundle of $f$.

Theorem 2.2 For every cohomology class $x \in H^{*}\left(M^{\times k}\right)$

$$
j_{k !}\left(i_{k}^{*}(x)\right)=\sum_{\alpha \in \mathrm{Eq}(k)} \Gamma_{|\alpha|}^{*}\left(1 \times f^{\times(|\alpha|-1)}\right) !\left(\left(\chi_{\Theta \in \alpha} A_{|\Theta|} e^{|\Theta|-1}\right) \cdot \Delta^{\alpha *}(x)\right),
$$

$$
f_{!}\left(j_{k !}\left(i_{k}^{*}(x)\right)\right)=\sum_{\alpha \in \mathrm{Eq}(k)} \Delta_{|\alpha|}^{*} f_{!}^{\times|\alpha|}\left(\left(\chi_{\Theta \in \alpha} A_{|\Theta|} e^{|\Theta|-1}\right) \cdot \Delta^{\alpha *}(x)\right) .
$$

In particular, if $x_{1}, \ldots, x_{k} \in H^{2 *}(M)$ (ie the $x_{i}$ have even dimension), then

$$
\begin{aligned}
j_{k !} i_{k}^{*}\left(x_{1} \times \cdots \times x_{k}\right)= & \sum_{\alpha \in \mathrm{Eq}(k)}\left(A_{|\alpha[1]|} e^{|\alpha[1]|-1} \prod_{i \in \alpha[1]} x_{i}\right) \\
\cdot & \prod_{\substack{\Theta \in \alpha \\
\Theta>\alpha[1]}}\left(A_{|\Theta|} f^{*} f_{!}\left(e^{|\Theta|-1} \prod_{i \in \Theta} x_{i}\right)\right), \\
f_{!} j_{k !} i_{k}^{*}\left(x_{1} \times \cdots \times x_{k}\right)= & \sum_{\alpha \in \mathrm{Eq}(k)} \prod_{\Theta \in \alpha}\left(A_{|\Theta|} f_{!}\left(e^{|\Theta|-1} \prod_{i \in \Theta} x_{i}\right)\right) .
\end{aligned}
$$

The two theorems together provide explicit formulas for the characteristic numbers and signature. We state only the signature formula: 
Corollary 2.3 The signature of the $k$-fold intersection manifold of $f$ is

$$
\begin{aligned}
\sigma\left(\Delta_{k}(f)\right)= & \sum_{l+\sum_{i=1}^{k-1} i l_{i}=k} \frac{(-1)^{k-1-\sum_{i=1}^{k-1} l_{i}}}{k \prod_{i=1}^{k-1} i_{i} l_{i} !} \\
& \cdot\left\langle L(M) e^{l-1} L(v)^{1-l} \prod_{i=1}^{k-1}\left(f^{*} f_{!}\left(e^{i-1} L(v)^{-i}\right)\right)^{l_{i}},[M]\right) \\
= & \sum_{\sum_{i=1}^{k} i l_{i}=k} \frac{(-1)^{k-\sum_{i=1}^{k} l_{i}}}{\prod_{i=1}^{k} i^{l_{i}} \cdot l_{i} !}\left\langle L(N) \prod_{i=1}^{k}\left(f_{!}\left(e^{i-1} L(v)^{-i}\right)\right)^{l_{i}},[N]\right\rangle
\end{aligned}
$$

where the indices $l_{i}$ run through the non-negative integers and the index $l$ runs through the positive integers.

So the general signature formula is similar to Hirzebruch's formula (1-2):

$$
\sigma\left(\Delta_{k}(f)\right)=\frac{1}{k !}\left\langle L(N) B_{k}(f),[N]\right\rangle,
$$

where $B_{k}(f)$ generalizes the product in (1-2):

$$
\begin{aligned}
B_{k}(f) & :=f_{!} j_{k} i_{k}^{*}\left(\bigcup_{i=1}^{k} L(v)^{-1}\right) \\
& =\sum_{\sum_{i=1}^{k} i l_{i}=k} \frac{k !(-1)^{k-\sum_{i=1}^{k} l_{i}}}{\prod_{i=1}^{k} i^{l_{i}} \cdot l_{i} !} \prod_{i=1}^{k}\left(f_{!}\left(e^{i-1} L(v)^{-i}\right)\right)^{l_{i}} .
\end{aligned}
$$

We now examine how this formula reduces to Hirzebruch's (1-2), ie the case when $M$ has several components.

Theorem 2.4 Let $M$ be the disjoint union of manifolds $M_{1}, \ldots, M_{l}$. Let $f_{i}$ denote the restriction of $f$ to $M_{i}$. Then we can compute $B_{k}(f)$ as

$$
B_{k}(f)=\sum_{k_{1}+\cdots+k_{l}=k} \frac{k !}{k_{1} ! \cdots k_{l} !} \prod_{i=1}^{l} B_{k_{i}}\left(f_{i}\right),
$$

where the $k_{i}$ run through the non-negative integers, and $B_{0}\left(f_{i}\right):=0$ by definition.

In particular, if the $M_{i}$ are embedded manifolds, then

$$
B_{l}(f)=\prod_{i=1}^{l} B_{1}\left(f_{i}\right)=\prod_{i=1}^{l} f_{i !}\left(L\left(v_{i}\right)^{-1}\right)
$$


where $v_{i}$ is the normal bundle of $f_{i}$.

Let $V_{i}$ be a 2 dimensional vector bundle over $N$. Let $M_{i}$ be the set of zeros of a transversal section of $V_{i}$. Let $f_{i}$ be the inclusion of $M_{i}$ into $N$. Then the normal bundle of $f_{i}$ is the restriction of $V_{i}$ to $M_{i}$, which means $v_{i}=f_{i}^{*}\left(V_{i}\right)$. Thus we have

$$
f_{i !}\left(L\left(v_{i}\right)^{-1}\right)=f_{i !}\left(f_{i}^{*}\left(L\left(V_{i}\right)^{-1}\right)\right)=e_{i} L\left(V_{i}\right)^{-1}
$$

where $e_{i}$ is the Euler class of $V_{i}$. Finally, Hirzebruch's formula (1-2) is obtained by combining (2-11), (2-14) and (2-15).

Theorem 2.2 will be proved in Section 4 and Section 5. The other results will be proved in Section 6.

\subsection{Special cases}

We present some special cases when the formulas above reduce to a product. We leave the proofs to Section 6.

$e, L(v)$ comes from $N$ If the cohomology classes $e$ and $L(v)$ are in the image of $f^{*}$, then the formulas simplify:

$$
j_{k !} i_{k}^{*}\left(f^{\times k}\right)^{*}(y)=\Delta_{k}^{*}\left(f^{\times k}\right)^{*}(y) \prod_{i=1}^{k-1}\left(f^{*} f_{!}(1)-i e\right)
$$

leading to

$$
\begin{aligned}
& \sigma\left(\Delta_{k}(f)\right)=\frac{1}{k !}\left\langle L(M) L(v)^{-(k-1)} \prod_{i=1}^{k-1}\left(f^{*} f_{!}(1)-i e\right),[M]\right\rangle \\
& p_{J}\left[\Delta_{k}(f)\right]=\frac{1}{k !}\left\langle\left(P(M) P(v)^{-(k-1)}\right)_{J} \prod_{i=1}^{k-1}\left(f^{*} f_{!}(1)-i e\right),[M]\right\rangle \\
& c_{J}\left[\Delta_{k}(f)\right]=\frac{1}{k !}\left\langle\left(C(M) C(v)^{-(k-1)}\right)_{J} \prod_{i=1}^{k-1}\left(f^{*} f_{!}(1)-i e\right),[M]\right\rangle .
\end{aligned}
$$

$\boldsymbol{e}=\mathbf{0}$ In case $e=0$, the only nonzero summand in (2-6) corresponds to $\alpha=0$.

$$
j_{k !} \circ i_{k}^{*}=\Gamma_{k}^{*} \circ\left(1 \times f^{\times(k-1)}\right) \text { ! }
$$


leading to

$$
\begin{aligned}
\sigma\left(\Delta_{k}(f)\right) & =\frac{1}{k !}\left\langle L(M)\left(f^{*} f_{!} L(v)^{-1}\right)^{k},[M]\right\rangle \\
& =\frac{1}{k !}\left\langle L(N)\left(f_{!}\left(L(v)^{-1}\right)\right)^{k},[N]\right\rangle \\
p_{J}\left[\Delta_{k}(f)\right] & =\frac{1}{k !}\left\langle\Delta_{k}^{*} f_{!}^{\times k}\left(P(M) \times \searrow_{i=2}^{k} P(v)^{-1}\right)_{J},[N]\right\rangle \\
c_{J}\left[\Delta_{k}(f)\right] & =\frac{1}{k !}\left\langle\Delta_{k}^{*} f_{!}^{\times k}\left(C(M) \times \searrow_{i=2}^{k} C(v)^{-1}\right)_{J},[N]\right\rangle .
\end{aligned}
$$

$f^{*} \boldsymbol{f}_{!}=\mathbf{0}$ If $f^{*} f_{!}=0$ (this is the case if $f$ is nullhomotopic and $n>0$ ) then the only nonzero summand in (2-8) corresponds to $\alpha=1$. Hence the formulas reduce to simple products:

$$
j_{k !} i_{k}^{*}\left(x_{1} \times \cdots \times x_{k}\right)=A_{k} e^{k-1}\left(x_{1} \cdots x_{k}\right)=(-1)^{k-1}(k-1) ! e^{k-1} x_{1} \cdots x_{k}
$$

leading to

$$
\begin{aligned}
\sigma\left(\Delta_{k}(f)\right) & =\frac{(-1)^{k-1}}{k}\left\langle e^{k-1} L(M) L(v)^{1-k},[M]\right\rangle \\
p_{J}\left[\Delta_{k}(f)\right] & =\frac{(-1)^{k-1}}{k}\left\langle e^{k-1}\left(P(M) P(v)^{1-k}\right)_{J},[M]\right\rangle \\
c_{J}\left[\Delta_{k}(f)\right] & =\frac{(-1)^{k-1}}{k}\left\langle e^{k-1}\left(C(M) C(v)^{1-k}\right)_{J},[M]\right\rangle .
\end{aligned}
$$

In particular, if $f$ is nullhomotopic, then $f^{*}(T N)$ is a trivial bundle and hence $L(v)^{-1}=L(M)$ and $P(v)^{-1}=P(M)$, so the formulas reduce to those in [3, Theorems 4 and 5] (up to minor notational differences), where these are claimed only when $N$ is $\mathbb{R}^{n}$ (which we can replace by the sphere $S^{n}$ if we want $N$ to be compact):

$$
\begin{aligned}
\sigma\left(\Delta_{k}(f)\right) & =\frac{(-1)^{k-1}}{k}\left\langle e^{k-1} L(M)^{k},[M]\right\rangle \\
p_{J}\left[\Delta_{k}(f)\right] & =\frac{(-1)^{k-1}}{k}\left\langle e^{k-1}\left(P(M)^{k}\right)_{J},[M]\right\rangle .
\end{aligned}
$$

This also corrects a typo (missing sign) in [3, Theorem 4]. 


\section{Sketch of proof}

From a technical point of view, the main result is formula (2-6). The other results easily follow from it, as we show in Section 6. In this section, we sketch the proof of formula (2-6).

Briefly, the proof splits into two parts: The first part (Section 4) studies the geometric situation to obtain formula (4-1). The second part is an algebraic rewrite of this formula to achieve our goal: (2-6).

In more detail, as in [3], the geometric idea is the description of the preimage of the (narrow) diagonal $\Delta_{k}(N)$ under $f^{\times k}$. Its components are parametrized by the equivalence relations on $k$ elements. The component belonging to an equivalence relation with $l$ equivalence classes is canonically isomorphic to the manifold of $l-$ tuple points of $f$. Ronga's Clean Intersection Theorem translates this geometric decomposition into formula (4-1).

The algebraic manipulation of (4-1) is guided by an interpretation of this formula as a power series equation: $G=F \circ H$, where $F$ collects the unknowns $j_{k !} i_{k}^{*}$. The power series $H$ turns out to be invertible so we can rewrite the formula as $F=G \circ H^{-1}$, which is just (2-6).

\section{From topology to algebra}

In this section we derive the recursion (4-1) on $j_{k !} i_{k}^{*}$.

Subcartesian diagram We will use Ronga's theorem on clean intersections [2, Proposition 2.2]. We recall the notion of clean intersection:

Definition 4.1 Two smooth functions $f: A \rightarrow M$ and $g: B \rightarrow M$ intersect cleanly if for every $a \in A$ and $b \in B$ such that $f(a)=g(b)$, there are local maps around $a$ of $A$, around $b$ of $B$ and around $f(a)=g(b)$ of $M$ such that both $f$ and $g$ are linear in these maps. It follows that

$$
Z:=\{(a, b) \in A \times B \mid f(a)=g(b)\}
$$

is a submanifold of $A \times B$, which we shall call the clean intersection of $f$ and $g$. The projections of $Z$ to $A$ and $B$ form a so called subcartesian diagram together with $f$ and $g$, see Figure 1. The excess vector bundle is the bundle $T M /(T A+T B)$ over $Z$, where we have omitted the obvious pullback functions in the notation as an abuse of language. 


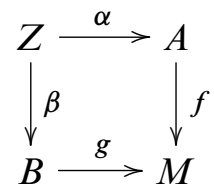

Figure 1: A subcartesian diagram

Ronga's theorem states a cohomological identity for subcartesian diagrams:

Theorem 4.2 (Clean Intersection Theorem [2, Proposition 2.2]) For every subcartesian diagram we have, using the notation of the above definition:

$$
g^{*}\left(f_{!}(x)\right)=\beta_{!}\left(e \cdot \alpha^{*}(x)\right) \quad\left(x \in H^{*}(A)\right),
$$

where $e$ is the Euler class of the excess bundle.

Main argument In this paragraph we apply the Clean Intersection Theorem to the maps $1 \times f^{\times(k-1)}$ and $\Gamma_{k}$ to obtain (4-1).

First, the clean intersection of the maps is the preimage of the image of $\Gamma_{k}$ under $1 \times f^{\times(k-1)}$, or, equivalently, the preimage of the diagonal $\Delta=\Delta^{k}(N)$ of $N^{\times k}$ under $f^{\times k}$. As in [3], this preimage is the disjoint union of closed submanifolds

$$
M_{\alpha}:=\left\{\left(x_{1}, \ldots, x_{k}\right) \in\left(f^{\times k}\right)^{-1}(\Delta) \mid x_{i}=x_{j} \Longleftrightarrow i \alpha j\right\},
$$

where $\alpha$ runs over the equivalence relations on $\{1, \ldots, k\}$. The manifold $M_{\alpha}$ is canonically isomorphic to $\widetilde{\Delta}_{|\alpha|}(f)$, and its inclusion into $M^{\times k}$ factors as

$$
M_{\alpha} \stackrel{i_{|\alpha|}}{\longrightarrow} M^{\times|\alpha|} \stackrel{\Delta^{\alpha}}{\longrightarrow} M^{\times k}
$$

Among these, $M_{0(k)}=\widetilde{\Delta}_{k}(f)$ is the manifold whose characteristic numbers we want to compute.

Second, we determine the maps in the subcartesian diagram. The map from $M_{\alpha}$ into $M^{\times k}$ is just the canonical embedding. The map from $M_{\alpha}$ to the factor $M$ of $\Gamma_{k}$ is the projection to the first coordinate.

All in all, the subcartesian diagram of $1 \times f^{\times(k-1)}$ and $\Gamma_{k}$ looks as the outer square of Figure 2. The inner square just explains some maps of the outer square.

We are going to compute the excess vector bundle. We will omit the pullback maps to simplify our notation since the context will always make it clear which map is missing. 


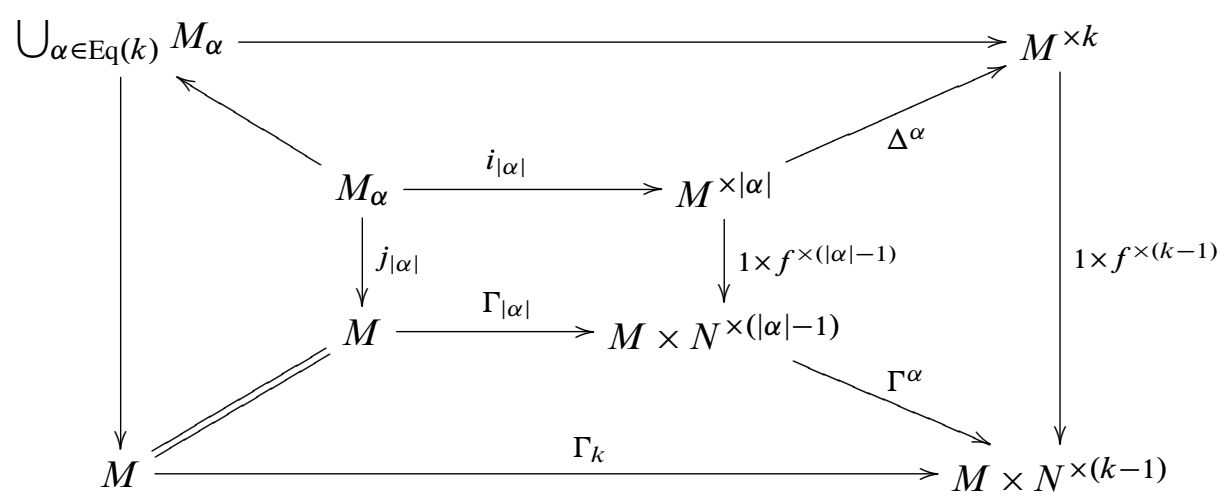

Figure 2: Multiple point manifolds of a generic immersion (top left) fitted into a subcartesian diagram (the outer square). The inner square is just for giving the maps of the outer square.

We fix an equivalence relation $\alpha$ on $\{1, \ldots, k\}$ and determine the excess vector bundle restricted to $M_{\alpha}$. Therefore we consider all vector bundles pulled back to $M_{\alpha}$.

Recall that the excess vector bundle is the factor of $T\left(M \times N^{\times(k-1)}\right)$ by $T M^{\times k}$ and $T M$. Notice that the inner square of the diagram is a transverse intersection because $f$ is generic, so the sum of $T M^{\times|\alpha|}$ (which is contained in $T M^{\times k}$ ) and $T M$ is $T\left(M \times N^{\times(|\alpha|-1)}\right)$. Thus the excess vector bundle is the factor of $T\left(M \times N^{\times(k-1)}\right)$ by $T M^{\times k}$ and $T\left(M \times N^{\times(|\alpha|-1)}\right)$.

At this point, we notice that the factor makes sense even on $M^{\times|\alpha|}$. Hence from now on we consider all vector bundles pulled back to $M^{\times|\alpha|}$.

Let $\Theta_{i}$ denote the $i$-th equivalence class of $\alpha$. We will write $l \xi$ for the direct sum of $l$ copies of a vector bundle $\xi$.

The embeddings of $T M^{\times k}$ and $T\left(M \times N^{\times(|\alpha|-1)}\right)$ into $T\left(M \times N^{\times(k-1)}\right)$ factor into the components of $M^{\times|\alpha|}$. For $i>1$ on the $i$-th component we have $\left|\Theta_{i}\right| T M$ and $T N$ embedded into $\left|\Theta_{i}\right| T N$. The bundle $T N$ is embedded diagonally, and the embedding of $\left|\Theta_{i}\right| T M$ is induced by $f$. Hence the factor is $\left(\left|\Theta_{i}\right|-1\right) v$, where $v$ is the normal bundle of $f$. The case of $i=1$ is similar.

Thus, the factor on $M^{\times|\alpha|}$ is $\left(\left|\Theta_{1}\right|-1\right) v \times \cdots \times\left(\left|\Theta_{l}\right|-1\right) v$ where $l:=|\alpha|$. Last, the excess vector bundle restricted to $M_{\alpha}$ is the restriction of this bundle from $M^{\times|\alpha|}$ to $M_{\alpha}$. 
Finally, applying the Clean Intersection Theorem (Theorem 4.2) to the diagram, one obtains:

$$
\Gamma_{k}^{*}\left(1 \times f^{\times(k-1)}\right) !(x)=\sum_{\alpha \in \mathrm{Eq}(k)} j_{|\alpha| !}\left(i_{|\alpha|}^{*}\left(\chi_{\Theta \in \alpha} e^{|\Theta|-1}\right) \cdot i_{|\alpha|}^{*} \Delta^{\alpha *} x\right) .
$$

Recall that $e=e(v)$ is the Euler class of $v$.

\section{The power series identity}

Now we have the recursion formula (4-1) on $j_{k !} i_{k}^{*}$. To make this recurrence relation transparent, we interpret it as a power series equality. Then our theorems will be reduced to routine calculations.

\subsection{Power series}

The general definition Power series are morphisms of the following category. Objects are sequences $A=\left(A_{k}\right)_{k=1}^{\infty}$ of modules. Let $A=\left(A_{k}\right)_{k=1}^{\infty}$ and $B=\left(B_{k}\right)_{k=1}^{\infty}$ be two sequences of modules. A morphism or power series $F$ from $A$ to $B$ is a collection of homomorphisms $\left(F_{\alpha}: A_{k} \rightarrow B_{|\alpha|} \mid \alpha \in \mathrm{Eq}(k), k=1, \ldots, \infty\right)$. Given two power series $F: A \rightarrow B$ and $G: B \rightarrow C$, we define their composite $G \circ F$ by

$$
(G \circ F)_{\alpha}=\sum_{\beta \leq \alpha} G_{\alpha / \beta} \circ F_{\beta} .
$$

Here $\beta$ and $\alpha$ are equivalence relations on the same set. The notation $\beta \leq \alpha$ means that every class of the equivalence relation $\alpha$ is a union of some classes of the equivalence relation $\beta$ (this is the usual ordering of equivalence relations). Thus $\alpha$ induces an equivalence relation $\alpha / \beta$ on the classes of $\beta$ : namely, those classes of $\beta$ are equivalent which belong to the same class of $\alpha$. There is a unique identification between the ordered set of classes of $\beta$ and the ordered set $\{1, \ldots,|\beta|\}$. Thus we may regard $\alpha / \beta$ as an equivalence relation on the latter set. This explains the notation in the above formula.

We leave the easy verifications of the axioms of category to the reader. The unit elements are of the form $E: A \rightarrow A$ defined as $E_{\alpha}=1$ if $\alpha=0 \in \mathrm{Eq}(k)$ for some $k$, and $E_{\alpha}=0$ for all other $\alpha$.

Classical examples Now we shall see that this definition is an extension of the usual definition of formal power series. Classically, given an analytic function $f: U \rightarrow$ $V$ between real vector spaces, its (exponential) power series is the sequence of its 
derivatives at a point $u$ of $U$. Let us denote by $\left(f_{k}: U^{\times k} \rightarrow V: k=1, \ldots, \infty\right)$ the $k$-th derivative of $f$ at $u$, it is a (symmetric) $k$-linear map. In our setting, this corresponds to $F$ : $\left(U^{\otimes k}\right)_{k=1}^{\infty} \rightarrow\left(V^{\otimes k}\right)_{k=1}^{\infty}$ defined by

$$
F_{\alpha}\left(u_{1} \otimes \cdots \otimes u_{k}\right):=\bigotimes_{\Theta \in \alpha} f_{|\Theta|}\left(u_{i}: i \in \Theta\right) .
$$

(The arguments of $f_{|\Theta|}$ are the elements $u_{i}: i \in \Theta$ in some order. The order does not matter since the function $f_{|\Theta|}$ is symmetric.) Our definition of composition generalizes the composition of usual power series, since Equation (5-1) is the generalization of the formula for the derivatives of a composite function.

By the above formula (5-2), we can define for all modules $U$ and $V$ and every sequence $\left(f_{k}: U^{\times k} \rightarrow V: k=1, \ldots, \infty\right)$ of symmetric multilinear maps a power series $F:\left(U^{\otimes k}\right)_{k=1}^{\infty} \rightarrow\left(V^{\otimes k}\right)_{k=1}^{\infty}$. Let us call power series of this form classical. They are clearly closed under composition. We will use classical power series for the $\mathbb{Z}[x]$-modules $U=V=\mathbb{Z}[x]$. In this special case, every $k$-linear function is of the form $f_{k}\left(x_{1}, \ldots, x_{k}\right)=a x_{1} \cdots x_{k}$ for some constant $a \in \mathbb{Z}[x]$.

\subsection{Power series in cohomology}

We are only interested in power series from the sequence of cohomology groups $\left(H^{*}\left(M^{\times k}\right)\right)_{k=1}^{\infty}$ to itself. We will denote this sequence by $\bar{H}^{*}(M)$.

Special series We want to map the monoid of classical power series of the $\mathbb{Z}[x]-$ module $\mathbb{Z}[x]$ to the monoid of power series of $\bar{H}^{*}(M)$. Substitution of an element $e \in H^{*}(M)$ for $x$ defines a ring homomorphism $\mathbb{Z}[x] \rightarrow H^{*}(M)$, which maps a polynomial $a$ into the cohomology class $a(e)$. Note that a classical power series of $\mathbb{Z}[x]$ is just a sequence $\left(a_{k}\right)_{k=1}^{\infty}$ of elements of $\mathbb{Z}[x]$. We map such a sequence to the power series $F$ defined by

$$
F_{\alpha}(y):=\left(\searrow_{\Theta \in \alpha} a_{|\Theta|}(e)\right) \cup \Delta^{\alpha *}(y) \quad\left(y \in H^{*}\left(M^{\times k}\right), \alpha \in \operatorname{Eq}(k)\right) .
$$

Note that the exponential power series of many real functions, like the exponential function exp and the natural logarithm $\ln$, have integer coefficients and hence are $\mathbb{Z} \rightarrow \mathbb{Z}$ and $\mathbb{Z}[x] \rightarrow \mathbb{Z}[x]$ series.

Solving the recursion We are now ready to analyze our recursion (4-1), which we repeat here in a slightly simpler form:

$$
\Gamma_{k}^{*}\left(1 \times f^{\times(k-1)}\right)_{!}(x)=\sum_{\alpha \in \mathrm{Eq}(k)}\left(j_{|\alpha| !} \circ i_{|\alpha|}^{*}\right)\left(\chi_{\Theta \in \alpha} e^{|\Theta|-1} \cdot \Delta^{\alpha *} x\right) .
$$


Our main observation is that the right-hand side is a special case of the composition formula (5-1). Let us form a power series from the unknown functions $j_{k !} \circ i_{k}^{*}$ :

$$
F_{\alpha}:= \begin{cases}j_{k !} \circ i_{k}^{*} & \text { if } \alpha=1(k) \text { for some } k \\ 0 & \text { otherwise. }\end{cases}
$$

Note that in the exponential power series expansion of the function

$$
H(x):=\frac{\exp (e x)-1}{e}=\sum_{k=1}^{\infty} \frac{e^{k-1}}{k !} x^{k}
$$

the coefficient of the $k$-th term is $e^{k-1}$, a polynomial in $e$. Therefore we may treat $H$ as a power series via (5-3) with $a_{k}=e^{k-1}$. So the right-hand side of (5-4) is just $(F \circ H)_{1(k)}$. Clearly, $(F \circ H)_{\alpha}=0$ for $\alpha \neq 1(k)$. Therefore, similarly to the definition of $F$, we can define a power series $G$ from the left-hand side of (5-4):

$$
G_{\alpha}:= \begin{cases}\Gamma_{k}^{*}\left(1 \times f^{\times(k-1)}\right) ! & \text { if } \alpha=1(k) \text { for some } k \\ 0 & \text { otherwise },\end{cases}
$$

so that our recursion (5-4) simply means

$$
G=F \circ H .
$$

The power series $H$ comes from an invertible function, and hence is invertible. The inverse is induced by the inverse function

$$
H^{-1}(y)=\frac{\ln (1+e y)}{e}=\sum_{k=1}^{\infty} \frac{(-1)^{k-1}(k-1) ! e^{k-1}}{k !} y^{k}=\sum_{k=1}^{\infty} \frac{A_{k} e^{k-1}}{k !},
$$

where $A_{k}:=(-1)^{k-1}(k-1)$ ! as declared in $(2-1)$. We see that the coefficients are polynomials in $e$ with integer coefficients and hence we may treat $H^{-1}$ as a power series from $\bar{H}^{*}(M)$ to itself via (5-3) with $a_{k}=A_{k} e^{k-1}$. Hence the solution of our recursion is $F=G \circ H^{-1}$, and this means

$$
j_{k !}\left(i_{k}^{*}(x)\right)=\sum_{\alpha \in \mathrm{Eq}(k)} \Gamma_{|\alpha|}^{*}\left(1 \times f^{\times(|\alpha|-1)}\right) !\left(\chi_{\Theta \in \alpha} A_{|\Theta|} e^{|\Theta|-1} \cdot \Delta^{\alpha *} x\right) .
$$

This is exactly Equation (2-6). Applying $f !$ to it and using the identity

$$
f_{!} \Gamma_{|\alpha|}^{*}\left(1 \times f^{\times(|\alpha|-1)}\right) !=\Delta^{\alpha *} f_{!}^{\times|\alpha|},
$$

we obtain (2-7). Substituting $x_{1} \times \cdots \times x_{k}$ for $x$ into these two formulas yield (2-8) and (2-9). We indicate below only how one can deduce (2-9) from (2-7). Recall that 
the $x_{i}$ are assumed to be even dimensional in Theorem 2.2, so no sign appears when we permute them.

$$
\begin{aligned}
\Delta^{\alpha *}\left(x_{1} \times \cdots \times x_{k}\right) & =\searrow_{\Theta \in \alpha} \prod_{i \in \Theta} x_{i} \\
\left(\chi_{\Theta \in \alpha} A_{|\Theta|} e^{|\Theta|-1}\right)\left(\searrow_{\Theta \in \alpha} \prod_{i \in \Theta} x_{i}\right) & =\searrow_{\Theta \in \alpha}\left(A_{|\Theta|} e^{|\Theta|-1} \prod_{i \in \Theta} x_{i}\right) \\
f_{!}^{\times|\alpha|}\left(\searrow_{\Theta \in \alpha} A_{|\Theta|} e^{|\Theta|-1} \prod_{i \in \Theta} x_{i}\right) & =\chi_{\Theta \in \alpha} A_{|\Theta|} f_{!}\left(e^{|\Theta|-1} \prod_{i \in \Theta} x_{i}\right)
\end{aligned}
$$

\section{Finishing the computation}

We have done the hard job in the previous sections. Now we derive the other results in Section 2 from Theorem 2.2 by direct computation.

\subsection{Proof of Theorem 2.1}

Recall, eg from [1, 5.1 and Theorem 8.2.2], that for every manifold $X$

$$
\begin{aligned}
\sigma(X) & =\langle L(X),[X]\rangle, \\
p_{J}[X] & =\left\langle P(X)_{J},[X]\right\rangle .
\end{aligned}
$$

We start by determining the normal bundle of $\widetilde{\Delta}_{k}(f)$ in $M^{\times k}$ using the diagram in Figure 3 , where $p_{k}:=f \circ j_{k}$.

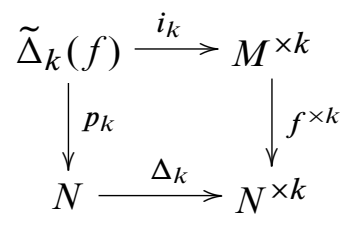

Figure 3: Multiple point manifold as part of a transverse intersection

Note that $\widetilde{\Delta}_{k}(f)$ is the transverse preimage of the diagonal of $N^{\times k}$ under $f^{\times k}$ at least in a neighbourhood of $\widetilde{\Delta}_{k}(f)$. So the normal bundle of $\widetilde{\Delta}_{k}(f)$ in $M^{\times k}$ is the pullback of the normal bundle of the diagonal in $N^{\times k}$ :

$$
v\left(i_{k}\right)=p_{k}^{*} \nu(\Delta)=p_{k}^{*}(\bigoplus T N)=i_{k}^{*}(1 \times \underbrace{f^{*} T N \times \cdots \times f^{*} T N}_{k-1}) .
$$


Hence one obtains for the Hirzebruch class of $\widetilde{\Delta}_{k}(f)$ :

$$
\begin{aligned}
& 2 L\left(\widetilde{\Delta}_{k}(f)\right)=2 i_{k}^{*}\left(L\left(M^{\times k}\right)\right) \cdot L\left(v\left(i_{k}\right)\right)^{-1} \\
& =2 i_{k}^{*}\left((L(M) \times \cdots \times L(M)) \cdot\left(1 \times L\left(f^{*} T N\right)^{-1} \times \cdots \times L\left(f^{*} T N\right)^{-1}\right)\right) \\
& =2 i_{k}^{*}\left(L(M) \times L(v)^{-1} \times \cdots \times L(v)^{-1}\right) .
\end{aligned}
$$

We have multiplied everything with 2 to get rid of eventual torsion parts. This has no consequence when computing the signature. We get by (6-1)

$$
\begin{aligned}
\sigma\left(\widetilde{\Delta}_{k}(f)\right) & =\left\langle i_{k}^{*}\left(L(M) \times L(v)^{-1} \times \cdots \times L(v)^{-1}\right),\left[\widetilde{\Delta}_{k}(f)\right]\right\rangle \\
& =\left\langle j_{k !} i_{k}^{*}\left(L(M) \times L(v)^{-1} \times \cdots \times L(v)^{-1}\right),[M]\right\rangle .
\end{aligned}
$$

This gives (2-2) of Theorem 2.1 since $\sigma\left(\Delta_{k}(f)\right)=\sigma\left(\widetilde{\Delta}_{k}(f)\right) / k$ !. Formula (2-3) is obtained by using $2 L(M)=2 f^{*}(L(N)) \cdot L(v)^{-1}$. The formulas (2-4) and (2-5) are proved similarly.

\subsection{Hirzebruch's virtual signature formula}

Proof of Corollary 2.3 The corollary is obtained by simply plugging Equation (2-8) directly into (2-2) and plugging (2-9) into (2-3). Substituting $x_{i}=L(v)^{-1}$ for all $i$ into (2-9), the summand corresponding to $\alpha$ will depend only on the number of elements of the classes of $\alpha$. Hence we can collect those summands together which are shown equal by this observation. Adding the collected terms, we obtain a new summation whose index will run through all tuples of non-negative integers $l_{1}, \ldots, l_{k}$ for which $\sum_{i=1}^{k} i l_{i}=k$, corresponding to the equivalence relations $\alpha$ with exactly $l_{i}$ pieces of $i$-element classes. There are exactly $k ! /\left(\prod_{i=1}^{k} i !^{l_{i}} \cdot l_{i} !\right)$ many such equivalence relations. Hence

$$
\begin{aligned}
f_{!} j_{k !} i_{k}^{*}\left(\chi_{i=1}^{k} L(v)^{-1}\right) & =\sum_{\sum_{i=1}^{k} i l_{i}=k} \frac{k !}{\prod_{i=1}^{k} i !_{i} \cdot l_{i} !} \prod_{i=1}^{k}\left(A_{i} f_{!}\left(e^{i-1} L(v)^{-i}\right)\right)^{l_{i}} \\
& =\sum_{\sum_{i=1}^{k} i l_{i}=k} \frac{k !(-1)^{k-\sum_{i=1}^{k} l_{i}}}{\prod_{i=1}^{k} i^{l_{i}} \cdot l_{i} !} \prod_{i=1}^{k}\left(f_{!}\left(e^{i-1} L(v)^{-i}\right)\right)^{l_{i}} .
\end{aligned}
$$

Recall from (2-1) that $A_{i}=(-1)^{i-1}(i-1)$ !, which is used in the second equation above.

This gives the second formula of Corollary 2.3. The first formula is obtained in a similar way using (2-8) but now the equivalence class of 1 is special. Therefore the summation runs through the tuples $\left(l, l_{1}, \ldots, l_{k-1}\right)$ corresponding to those equivalence 
relations for which the class of 1 has $l$ elements and there are exactly $l_{i}$ classes with $i$ elements which does not contain 1 . The number of such equivalence relations is $(k-1) ! /\left((l-1) ! \prod_{i=1}^{k} i !^{l_{i}} \cdot l_{i} !\right)$. Therefore

$$
\begin{gathered}
j_{k !} i_{k}^{*}\left(L(M) \times \chi_{i=2}^{k} L(v)^{-1}\right)=\sum_{l+\sum_{i=1}^{k-1} i l_{i}=k} \frac{(k-1) !}{(l-1) ! \prod_{i=1}^{k-1} i l_{i} l_{l} !} A_{l} e^{l-1} L(M) \\
\cdot L(v)^{1-l} \prod_{i=1}^{k-1}\left(A_{i} f^{*} f_{!}\left(e^{i-1} L(v)^{-i}\right)\right)^{l_{i}} \\
=\sum_{l+\sum_{i=1}^{k-1} i l_{i}=k} \frac{(k-1) !(-1)^{k-1-\sum_{i=1}^{k} l_{i}}}{\prod_{i=1}^{k-1} i^{l_{i}} \cdot l_{i} !} L(M) e^{l-1} \\
\cdot L(v)^{1-l} \prod_{i=1}^{k-1}\left(f^{*} f_{!}\left(e^{i-1} L(v)^{-i}\right)\right)^{l_{i}} .
\end{gathered}
$$

The exponent of $(-1)$ in both formulas of the Corollary is the difference between $k$ and the number of equivalence classes.

Proof of Theorem 2.4 Now we examine the case when $M$ is a disjoint union of manifolds $M_{1}, \ldots, M_{l}$. Then the cartesian power $M^{\times k}$ is the disjoint union of products $M_{l_{1}} \times \cdots \times M_{l_{k}}$ for all $1 \leq l_{1}, \ldots, l_{k} \leq l$. This decomposition also decomposes $\widetilde{\Delta}_{k}(f)$, which leads to a decomposition of $j_{k !} i_{k}^{*}$, and hence $B_{k}(f)$, into a sum. We are gong to determine the summands.

Therefore, let us fix a tuple $\left(l_{1}, \ldots, l_{k}\right)$. Let $\tilde{j}_{k}$ denote the restriction of $j_{k}$ to $\widetilde{\Delta}_{k}(f) \cap$ $M_{l_{1}} \times \cdots \times M_{l_{k}}$, and, similarly, let $\widetilde{i_{k}}$ denote the restriction of $i_{k}$.

Let $k_{t}$ be the multiplicity of $t$ in the tuple, so that $M_{l_{1}} \times \cdots \times M_{l_{k}} \cong \times_{t=1}^{l} M_{t}^{\times k_{t}}$ by a permutation of coordinates. Let $s$ denote the inclusion of this space into $M^{\times k}$.

Under this identification, $\tilde{\Delta}_{k}(f) \cap M_{l_{1}} \times \cdots \times M_{l_{k}}$ is clearly a subspace of the product $\chi_{t=1}^{l} \widetilde{\Delta}_{k_{t}}\left(f_{t}\right)$. Actually, it is the preimage of the diagonal of $N^{\times l}$ under $X_{t=1}^{l}\left(f \circ j_{k_{t}}^{\left(f_{t}\right)}\right)$, so the square in Figure 4 is a transverse intersection since $f$ is generic. In the diagram $p$ denotes the obvious inclusion map.

By transversality, we have (eg as a special case of Theorem 4.2):

$$
f_{!} \tilde{j}_{k !} p^{*}=\Delta_{l}^{*}\left(\chi_{t=1}^{l} f \circ j_{k_{t}}^{\left(f_{t}\right)}\right)_{!}
$$




$$
\begin{aligned}
& \widetilde{\Delta}_{k}(f) \cap \chi_{t=1}^{l} M_{l_{t}} \stackrel{p}{\longrightarrow} \chi_{t=1}^{l} \tilde{\Delta}_{k_{t}}\left(f_{t}\right) \stackrel{\Upsilon_{t=1}^{l} i_{k_{t}}^{\left(f_{t}\right)}}{\longrightarrow} \chi_{t=1}^{l} M_{t}^{\times k_{t}} \stackrel{s}{\longrightarrow} M^{\times k} \\
& \begin{array}{l}
\downarrow f \circ \tilde{j_{k}} \\
N \stackrel{\Delta_{l}}{\longrightarrow} X_{t=1}^{l} N
\end{array}
\end{aligned}
$$

Figure 4: Decompositions of multiple point manifold for immersion of a nonconnected manifold

The composition of the top row is $\tilde{i_{k}}$, so we obtain

$$
f_{!} \tilde{j}_{k !} \tilde{i}_{k}^{*}=f_{!} \tilde{j}_{k !} p^{*}\left(\searrow_{t=1}^{l} i_{k_{t}}^{\left(f_{t}\right)}\right)^{*} s^{*}=\Delta_{l}^{*}\left(\searrow_{t=1}^{l} f \circ j_{k_{t}}^{\left(f_{t}\right)}\right)_{!}\left(\searrow_{t=1}^{l} i_{k_{t}}^{\left(f_{t}\right)}\right)^{*} s^{*} .
$$

Evaluating the expression at $\chi_{i=1}^{k} L(v)^{-1}$, we obtain the summand of $B_{k}(f)$ corresponding to $\left(l_{1}, \ldots, l_{k}\right)$. We write $v_{t}$ for the normal bundle of $f_{t}$, which is the restriction of $v$ to $M_{t}$.

$$
\begin{aligned}
& \Delta_{l}^{*}\left(\searrow_{t=1}^{l} f \circ j_{k_{t}}^{\left(f_{t}\right)}\right)_{!}\left(\searrow_{t=1}^{l} i_{k_{t}}^{\left(f_{t}\right)}\right)^{*} s^{*}\left(\searrow_{i=1}^{k} L(v)^{-1}\right) \\
& =\Delta_{l}^{*}\left(\searrow_{t=1}^{l} f \circ j_{k_{t}}^{\left(f_{t}\right)}\right),\left(\searrow_{t=1}^{l} i_{k_{t}}^{\left(f_{t}\right)}\right)^{*}\left(\searrow_{t=1}^{l}\left(L\left(v_{t}\right)^{-1}\right)^{\times k_{t}}\right) \\
& =\prod_{t=1}^{l} f_{t !} j_{k_{t} !}^{\left(f_{t}\right)}\left(i_{k_{t}}^{\left(f_{t}\right)}\right)^{*}\left(L\left(v_{t}\right)^{-1}\right)^{\times k_{t}}=\prod_{t=1}^{l} B_{k_{t}}\left(f_{t}\right) .
\end{aligned}
$$

The class $B_{k}(f)$ is the sum of the last expression for all tuples $\left(l_{1}, \ldots, l_{k}\right)$, which leads to $(2-13)$. Note that a tuple $k_{1}, \ldots, k_{l}$ appears exactly for $k ! /\left(k_{1} ! \cdots k_{l} !\right)$ many tuples $\left(l_{1}, \ldots, l_{k}\right)$.

\subsection{Proof of the special cases}

Most of the special cases in Subsection 2.3 follow easily from the general formula, since almost all summands become zero in (2-6). Let us examine Equation (2-6) in more detail. If $e=0$ then all summands containing $e$ become zero and hence only the summand corresponding to $\alpha=1$ can be nonzero. If $f^{*} f_{!}=0$ then we use (2-8) and see that the only summand which does not contain $f^{*} f$ ! corresponds to $\alpha=0$, and hence the other summands are zero. 
Finally, the case " $\boldsymbol{e}, \boldsymbol{L}(\boldsymbol{v})$ comes from $\boldsymbol{N}$ " requires more calculations. We will use power series again to save some computations. Let us evaluate (2-6) at $x=1$.

$$
j_{k !}(1)=\sum_{\alpha \in \mathrm{Eq}(k)}\left(\prod_{\Theta \in \alpha} A_{|\Theta|} e^{|\Theta|-1}\right)\left(f^{*} f_{!}(1)\right)^{|\alpha|-1}=\partial_{3}^{k} q\left(e, f^{*} f_{!}(1), 0\right),
$$

where the sum is again a special case of the formula of composition of power series (or higher order derivatives). Namely, the two functions we compose are

$$
\begin{aligned}
g(y, z) & :=\frac{\exp y z-1}{y} \\
h(x, z) & :=\frac{\ln (1+x z)}{x}
\end{aligned}
$$

and $q$ is their composition:

$$
q(x, y, z):=g(y, h(x, z))=\frac{1}{y}\left(\exp \left(y \frac{\ln (1+x z)}{x}\right)-1\right) .
$$

Now we find the terms of the power series $q$ using ordinary power series. Recall that

$$
\exp (t \ln (1+x))=(1+x)^{t}=\sum_{n=0}^{\infty}\left(\begin{array}{l}
t \\
n
\end{array}\right) x^{n}
$$

Substituting $y / x$ for $t$ and $x z$ for $x$ this becomes

$$
1+y q(x, y, z)=\exp \left(y \frac{\ln (1+x z)}{x}\right)=\sum_{n=0}^{\infty}\left(\begin{array}{c}
y / x \\
n
\end{array}\right) x^{n} z^{n} .
$$

Thus the $n$-th partial derivative of $q$ in its third variable $z$ is

$$
\partial_{3}^{n} q(x, y, 0)=\frac{n !}{y}\left(\begin{array}{c}
y / x \\
n
\end{array}\right) x^{n}=\prod_{i=1}^{n-1}(y-i x) .
$$

Plugging this into (6-5), we obtain

$$
\begin{aligned}
j_{k !}(1) & =\prod_{i=1}^{k-1}\left(f^{*} f_{!}(1)-i e\right), \\
j_{k !} i_{k}^{*}\left(f^{\times k}\right)^{*}(y) & =\Delta_{k}^{*}\left(f^{\times k}\right)^{*}(y) j_{k !}(1)=\Delta_{k}^{*}\left(f^{\times k}\right)^{*}(y) \prod_{i=1}^{k-1}\left(f^{*} f_{!}(1)-i e\right) .
\end{aligned}
$$

The last formula is exactly (2-16), from which (2-17), (2-18) and (2-19) are straightforward. 


\section{References}

[1] F Hirzebruch, Topological methods in algebraic geometry, Classics in Math., Springer, Berlin (1995) MR1335917 Translated from the German and Appendix One by R LE Schwarzenberger, with a preface to the third English edition by the author and Schwarzenberger, Appendix Two by A Borel; Reprint of the 1978 edition

[2] F Ronga, On multiple points of smooth immersions, Comment. Math. Helv. 55 (1980) 521-527 MR604710

[3] A Szúcs, On the multiple points of immersions in Euclidean spaces, Proc. Amer. Math. Soc. 126 (1998) 1873-1882 MR1451830

Alfréd Rényi Institute of Mathematics, Hungarian Academy of Sciences

Budapest, Reáltanoda u 13-15, 1053, Hungary

braung at renyi.hu

http://www.renyi.hu/ braung/

Received: 27 June $2005 \quad$ Revised: 19 April 2006 